\title{
Trends in Hysterectomy Incidence Rates During 2000-20I5 in Denmark: Shifting from Abdominal to Minimally Invasive Surgical Procedures
}

\author{
Kathrine Dyhr Lycke (iD ${ }^{1,2}$ \\ Johnny Kahlert ${ }^{3}$ \\ Rikke Damgaard ${ }^{1,2}$ \\ Ole Mogensen ${ }^{2,4}$ \\ Anne Hammer ${ }^{1,2,4}$ \\ 'Department of Obstetrics and \\ Gynecology, NIDO | Denmark, Gødstrup \\ Hospital, Herning, Denmark; \\ ${ }^{2}$ Department of Clinical Medicine, \\ Aarhus University, Aarhus, Denmark; \\ ${ }^{3}$ Department of Clinical Epidemiology, \\ Aarhus University Hospital, Aarhus, \\ Denmark; ${ }^{4}$ Department of Obstetrics \\ and Gynecology, Aarhus University \\ Hospital, Aarhus, Denmark
}

\begin{abstract}
Background: Hysterectomy (removal of the uterus) is a common surgical procedure in gynecology. Although minimally invasive surgical procedures have been introduced, hysterectomy is still associated with risk of short- and long-term complications. Given that hysterectomized women are no longer at risk of either hysterectomy or being diagnosed with endometrial or cervical cancer, it is important to describe trends in hysterectomy rates. Objective: To describe trends in hysterectomy incidence rates overall and stratified by age, indication, and procedure.
\end{abstract}

Methods: Nationwide population-based cohort study using Danish national registries, 2000-2015, was conducted. We calculated the overall hysterectomy-corrected and agestandardized incidence rates of hysterectomy among women $\geq 20$ years old. Incidence rates were stratified by age group, indication, and surgical procedure. We performed trend analyses using Joinpoint regression, thereby estimating the average annual percentage change (AAPC).

Results: A total of 98,484 women had a hysterectomy during the study period, corresponding to an overall age-standardized, hysterectomy-corrected hysterectomy incidence rate (SIR) of 351.1 per 100,000 person-years $(95 \%$ CI 348.9;353.3). SIR of hysterectomy declined over time (AAPC $-1.4 ; 95 \% \mathrm{CI}-1.9 ;-1.0$ ), which was driven by a decline in rates of benign hysterectomy (AAPC $-2.1 ; 95 \%$ CI $-2.7 ;-1.6$ ). Irrespective of indication, rates of abdominal hysterectomy declined substantially during the study period and were surpassed by rates of minimally invasive procedures (ie, laparoscopy and robot-assisted laparoscopy) in 2013.

Conclusion: Hysterectomy-corrected incidence rates of benign hysterectomy declined over time. Irrespective of indication, we observed a shift in surgical procedure over time, from abdominal hysterectomy to minimally invasive surgical procedures.

Keywords: hysterectomy, incidence, surgical procedure, indication, epidemiology

\section{Introduction}

Hysterectomy is one of the most common surgical procedures in gynecology, and it may be performed due to benign or malignant disease. Hysterectomy includes the removal of the uterine corpus and typically the cervix as well. Often, hysterectomy also includes the removal of both fallopian tubes, as this may reduce subsequent risk of ovarian cancer. ${ }^{1-3}$ Hysterectomy may also include the removal of both ovaries. $^{2,3}$ The procedure may be performed through an incision in the abdominal wall, by laparoscopy (with or without robot-assistance), or vaginally. Despite the introduction of minimally invasive procedures in the past decades, ${ }^{4,5}$ hysterectomy
Correspondence: Kathrine Dyhr Lycke Department of Obstetrics and Gynecology, NIDO | Denmark, Gødstrup Hospital, GI. Landevej 6I, Herning, DK7400, Denmark

Email katlyc@clin.au.dk 
is still associated with risk of surgical complications, including bleeding, infection, and damage to the bladder and bowels. ${ }^{6}$ Moreover, studies show that women undergoing hysterectomy are at increased risk of physical impairment, ${ }^{7}$ pain, ${ }^{8,9}$ depression, ${ }^{10}$ and cardiovascular disease. $^{11}$

Globally, hysterectomy incidence rates vary across countries, with rates being two-fold higher in Australia and the United States compared to the Scandinavian countries. $^{5,12-14}$ The overall hysterectomy incidence is important for proper calculation of incidence rates of cervical and endometrial cancer, as only women with an intact cervix and uterine corpus, respectively, are at risk of these diseases. ${ }^{2,15-20}$ Similarly, only women with an intact uterus are at risk of undergoing a hysterectomy. ${ }^{13,21}$ Most previous studies have reported trends in uncorrected hysterectomy incidence rates; few have reported hysterectomy incidence rates after correcting for hysterectomy. ${ }^{13,21}$ However, in previous studies, correction for hysterectomy was based on surveys and, therefore, results may be subject to recall bias.

Using individual-level data from high-quality nationwide registries, we provide an update on temporal trends in hysterectomy incidence rates in Denmark after correcting for hysterectomy prevalence, overall and stratified by age, indication, and surgical procedure.

\section{Materials and Methods}

\section{Study Population and Design}

We conducted a nationwide population-based cohort study in Denmark from 2000 to 2015. In Denmark, the health care system is tax-funded and all citizens have free and equal access to general practitioners and public hospitals. Using the Danish civil registration number (CPR-number), a unique code assigned to every resident at birth or immigration, it is possible to link prospectively collected individual-level data from Danish nationwide registries. ${ }^{22}$ The CPR-number is registered in the Civil Registration System and contains information on date of birth, emigration status, vital status, and date of vital status. The Civil Registration System has virtually complete follow-up. ${ }^{22}$ Estimates in the present study are based on the adult female population residing in Denmark at some point during the study period, 2000-2015 ( $\mathrm{N}=3.55$ million). Women $<20$ years were excluded because the risk of hysterectomy was extremely low.

\section{Data Sources}

Information on hysterectomy was collected from the Danish National Registry of Patients (DNRP). DNRP was established in 1977 and holds information on all nonpsychiatric contacts at private and public hospitals in Denmark, including all in-patient admissions. Data from each contact include the patient's CPR number, type of contact (in-patient, out-patient, emergency room), treatment, surgical procedures, dates of admission and discharge, and up to 20 diagnoses coded according to the International Classification of Disease 8th edition (ICD-8) until 1993 and ICD-10 since 1994. ${ }^{23}$

As summarized in Supplementary Table 1, we categorized surgical procedures according to the procedure codes registered in DNRP; laparoscopy, robot-assisted laparoscopy, abdominal, vaginal, supravaginal (ie, leaving the cervix intact), and radical. A procedure was considered robot-assisted if it contained the additional procedure code (KZXX0), irrespective of the main procedure code. Of note, most robot-assisted hysterectomies had laparoscopy as the main procedure code (98\%), suggestting a low conversion rate. We collected information on the primary diagnosis code for all patients to determine whether the indication for the procedure was benign or malignant (Supplementary Table 2). In the present study, borderline ovarian tumors (DD391A), cervical neoplasia (DN87*), carcinoma in situ (DD0*), and atypical endometrial hyperplasia (DD0*) were classified as malignant. The rationale behind this decision was that these diagnoses are associated with increased risk of cancer compared to benign diagnoses (eg, abnormal uterine bleeding) and, as a result, these diagnoses may be managed with the corresponding malignant diagnoses, particularly borderline ovarian tumors and atypical hyperplasia.

\section{Statistical Analyses}

We tabulated the distribution of all hysterectomies in the study period by age group, calendar year, type of procedure, and indication. We calculated the uncorrected and hysterectomy-corrected incidence rates (IR) and agestandardized incidence rates (SIR) of hysterectomy per 100,000 person-years, overall and stratified by age group (20-39, 40-54, 55+), surgical procedure (laparoscopy, robot-assisted laparoscopy, abdominal, vaginal, supravaginal, radical), and indication (benign, malignant). We conducted a sensitivity analysis in which the premalignant 
diagnosis codes were classified as benign instead of malignant.

Incidence rates were age-standardized to the US 2010 female population. For the uncorrected estimates, women contributed time at-risk from January 1, 2000 or age 20 to date of hysterectomy, emigration, death, or December 31, 2015, whichever came first. For calculation of hysterectomy-corrected IR of hysterectomy, women were excluded from the population at-risk denominator if they had undergone a hysterectomy between January 1, 1977 through December 31, 1999. Since DNRP was established in 1977, some women may have undergone hysterectomy before 1977. Therefore, we made an additional correction considering the probability of hysterectomy prior to 1977 for women aged 20 or older, as described in details elsewhere. ${ }^{16,18}$ The lifetime cumulative probability of hysterectomy used for hysterectomy-correction was performed using STATA version 15.1 (StataCorp. College Station, TX), whereas the remaining statistical analyses were undertaken using SAS, v. 9.4 (SAS Institute, Cary, NC). In this study, we mainly present hysterectomy-corrected IR and SIR, unless otherwise stated. Uncorrected rates are reported in Supplementary Table 3.

To estimate temporal trends in hysterectomy incidence rates during the study period, we calculated the average annual percentage change (AAPC) using the National Cancer Institute's Joinpoint Regression Program, version $4.8 .^{24}$

\section{Results}

During the study period, 2000-2015, a total of 98,484 hysterectomies were registered, $74 \%$ of which were performed due to benign disease and $26 \%$ due to malignant disease (Table 1). The annual number of hysterectomies declined by $12 \%$, from 6,252 hysterectomies in 2000 to 5,530 in 2015. Median age at hysterectomy was 49 years overall (interquartile range, IQR 43;60); 47 years (IQR 43;54) for women having a benign hysterectomy, and 61 years (IQR 50;70) for women undergoing hysterectomy due to malignant disease. Two-thirds of benign hysterectomies were performed among women aged 40-54, whereas two-thirds of malignant hysterectomies were performed among women aged 55+.

As seen in Table 1, 78 of benign hysterectomies were classified as radical (of 2,152 radical hysterectomies in total). Because radical hysterectomy is a procedure performed due to cervical cancer, we
Table I Number and Percentage of Hysterectomies, Stratified by Age, Calendar Year, and Surgical Procedure

\begin{tabular}{|c|c|c|c|}
\hline & $\begin{array}{l}\text { Total } \\
\text { n (\%) }\end{array}$ & $\begin{array}{l}\text { Benign } \\
\text { n (\%) }\end{array}$ & $\begin{array}{l}\text { Malignant } \\
\text { n (\%) }\end{array}$ \\
\hline Total & $98,484(100)$ & $73,359(100)$ & $25,125(100)$ \\
\hline \multicolumn{4}{|l|}{ Age } \\
\hline $20-39$ & II,424 (II.6) & $9,237(12.6)$ & $2,187(8.7)$ \\
\hline $40-54$ & $53,291(54.1)$ & $46,936(64.0)$ & $6,355(25.3)$ \\
\hline $55+$ & $33,769(34.3)$ & $17,186(23.4)$ & $16,583(66.0)$ \\
\hline \multicolumn{4}{|l|}{ Calendar year } \\
\hline 2000 & $6,252(6.3)$ & 4,831 (6.6) & $|, 42|(5.7)$ \\
\hline 2001 & $6.680(6.8)$ & $5,098(6.9)$ & I,582 (6.3) \\
\hline 2002 & $6,980(7.1)$ & $5,480(7.5)$ & I,500 (6.0) \\
\hline 2003 & $6,792(6.9)$ & $5,287(7.2)$ & I,475 (5.9) \\
\hline 2004 & $6,229(6.3)$ & $4,760(6.5)$ & I,469 (5.8) \\
\hline 2005 & $6.236(6.3)$ & $4,75 \mid(6.5)$ & I,485 (5.9) \\
\hline 2006 & $6,138(6.2)$ & $4,650(6.4)$ & I,488 (5.9) \\
\hline 2007 & $6,187(6.3)$ & $4,670(6.4)$ & $1,517(6.0)$ \\
\hline 2008 & $5,832(5.9)$ & $4,257(5.8)$ & I,575 (6.3) \\
\hline 2009 & $6,279(6.4)$ & $4,636(6.3)$ & $\mathrm{I}, 643(6.5)$ \\
\hline 2010 & $5,489(5.9)$ & $4,310(5.9)$ & I,539 (6.I) \\
\hline 2011 & $6,100(6.2)$ & $4,439(6.1)$ & $\mathrm{I}, 66 \mathrm{I}(6.6)$ \\
\hline 2012 & $5,787(5.9)$ & $4,123(5.6)$ & I,664 (6.6) \\
\hline 2013 & $5,774(5.9)$ & $4.063(5.5)$ & $|, 7| \mid(6.8)$ \\
\hline 2014 & $5,869(6,0)$ & $4, I 44(5.6)$ & I,725 (6.9) \\
\hline 2015 & $5,530(5.6)$ & $3,860(5.3)$ & $1,670(6.6)$ \\
\hline \multicolumn{4}{|l|}{$\begin{array}{l}\text { Surgical } \\
\text { procedure }\end{array}$} \\
\hline Laparoscopic & $12,249(12.4)$ & $9,732(13.3)$ & $2,517(10.0)$ \\
\hline Robot-assisted & 3,449 (3.5) & $868(1.2)$ & $2,58 \mathrm{I}(10.2)$ \\
\hline Abdominal & $49,208(50.0)$ & $32,940(44.9)$ & $16,268(64.5)$ \\
\hline Vaginal & $21,890(22.2)$ & $20,697(28.2)$ & I, $193(4.7)$ \\
\hline Supravaginal & $9,536(9.7)$ & $9,044(12.3)$ & $492(2.0)$ \\
\hline Radical & $2,152(2.2)$ & $78(0.1)$ & $2,074(8.2)$ \\
\hline
\end{tabular}

considered the 78 cases misclassified and excluded them from further analyses (ie IR and SIR, stratified by indication and procedure).

The overall hysterectomy-corrected SIR of hysterectomy was 351.1 per 100,000 person-years (95\% CI 348.9; 353.3) (Table 2). Age-standardized incidence rates of benign hysterectomy were nearly three-fold higher than rates of malignant hysterectomy (261.7 vs 89.1 per 100,000 person-years). When stratifying by age at hysterectomy, the highest IRs were found among women aged 40-54 followed by women aged 55+, whilst women aged 20-39 had the lowest IRs (Table 2). With respect to surgical procedure, SIR of abdominal hysterectomy was highest, followed by vaginal and laparoscopic hysterectomy (Table 2). 
Table 2 Overall Hysterectomy-Corrected, Age-Standardized (US 2010 Female Population) Incidence Rate per 100,000 Person-Years, Stratified by Age, Indication, and Surgical Procedure

\begin{tabular}{|c|c|c|c|}
\hline & \multirow[t]{2}{*}{$\mathbf{n}$} & \multicolumn{2}{|l|}{ Corrected } \\
\hline & & IR (95\% Cl) & SIR (95\% CI) \\
\hline Total & 98,484 & $343.8(341.6 ; 345.9)$ & $35 \mathrm{I} . \mathrm{l}(348.9 ; 353.3)$ \\
\hline \multicolumn{4}{|l|}{ Age } \\
\hline $20-39$ & II,424 & $107.8(105.9 ; 109.8)$ & NA \\
\hline $40-54$ & 53,291 & $674.0(668.3 ; 679.7)$ & NA \\
\hline $55+$ & 33,769 & $332.9(329.3 ; 336.4)$ & NA \\
\hline \multicolumn{4}{|l|}{ Indication } \\
\hline Benign & 73,281 & $255.8(254.0 ; 257.7)$ & $261.7(259.8 ; 263.6)$ \\
\hline Malignant & 25,125 & $87.7(86.6 ; 88.8)$ & $89.1(88.0 ; 90.2)$ \\
\hline \multicolumn{4}{|l|}{$\begin{array}{l}\text { Surgical } \\
\text { procedure }\end{array}$} \\
\hline Laparoscopic & 12,249 & $42.8(42.0 ; 43.5)$ & $43.6(42.8 ; 44.4)$ \\
\hline Robot-assisted & 3,449 & $12.0(\mid 1.6 ; 12.4)$ & $12.2(11.8 ; 12.6)$ \\
\hline Abdominal & 49,208 & I7I.8 (I70.3;173.3) & I75.6 (|74.1;177.2) \\
\hline Vaginal & 21,890 & $76.4(75.4 ; 77.4)$ & $77.9(76.8 ; 78.9)$ \\
\hline Supravaginal & 9,536 & $33.3(32.6 ; 34.0)$ & $34.2(33.5 ; 34.8)$ \\
\hline Radical & 2,074 & $7.2(6.9 ; 7.6)$ & $7.3(7.0 ; 7.6)$ \\
\hline
\end{tabular}

Abbreviation: NA, not applicable.

\section{Temporal Trends in Hysterectomy Incidence Rates}

During the study period, overall hysterectomy-corrected SIR of hysterectomy declined from 362.9 per 100,000 person-years (95\% CI 353.0;372.04) in 2000 to 307.1 per $100,000(95 \%$ CI 299.1; 315.3) in 2015 (AAPC -1.4, 95\% CI -1.9; -1.0) (Figure 1 and Supplementary Table 3). The decline was attributed to a decline in SIR of benign hysterectomy, from 279.2 (95\% CI 271.4; 287.2) in 2000 to 216.2 (95\% CI 209.4; 223.1) in 2015 (AAPC -2.1; 95\% CI -2.7 ; -1.6) (Figure 2). In contrast, SIR of malignant hysterectomy increased slightly, from 83.1 (95\% CI 78.8; 87.5 ) in 2000 to $90.9(86.6 ; 95.4)$ in 2015 (AAPC 0.6; 95\% CI 0.2 ; 1.0) (Figure 3 ). We performed a sensitivity analysis in which premalignant diagnosis codes were classified as benign instead of malignant. This resulted in an overall increase in SIR of benign hysterectomy, whereas the temporal trend was attenuated (AAPC - 1.7; 95\% CI -2.3; -1.2) (Supplementary Figure 1). SIR of malignant hysterectomy was lower in our sensitivity analysis compared to our main analysis, and the previously observed increase in SIR of malignant hysterectomy over calendar time was no longer observed (AAPC -0.2; 95\% CI -0.6; 0.2 ). With respect to age, SIR of hysterectomy declined among women aged 20-39 years (AAPC - 1.8; 95\% CI $-2.6 ;-1.0$ ) and women aged 40-54 (AAPC - 2.0; 95\% CI $-2.5 ;-1.5)$, whereas rates remained unchanged for women aged 55 years and older (AAPC $-0.4 ; 95 \%$ CI $-0.9 ; 0.2$ ) (Supplementary Table 3).

\section{Trends in Type of Procedure}

During the study period, we observed changes in SIR by surgical procedure (Figure 1). SIR of abdominal hysterectomy declined by $64 \%$, from 210.5 per 100,000 person-years in 2000 to 76.2 per 100,000 person-years in 2015 (AAPC -5.6; $95 \%$ CI $-7.5 ;-3.7)$, and SIR of supravaginal hysterectomy declined by $90 \%$, from 72.3 per 100,000 person-years to 7.4 per 100,000 person-years (AAPC -12.3 ; 95\% CI -14.1; -10.5). In contrast, SIR of laparoscopic hysterectomy increased 8-fold, from 16.9 per 100,000 person-years to 132.8 per 100,000 person-years (AAPC 21.1; 95\% CI 16.5; 25.9), and robot-assisted hysterectomy increased 70-fold, from 0.7 per 100,000 person-years in 2008 to 48.8 per 100,000 person-years in 2015 (AAPC 43.6; 95\% CI $20.2 ; 71.5)$.

\section{Trends in Type of Procedure by Indication}

When stratifying by procedure and indication, similar trends were observed for SIR of benign hysterectomy, with a major reduction in rates of abdominal hysterectomy (AAPC -5.9; 95\% CI -7.8; -4.0) and supravaginal hysterectomy (AAPC $-12.1 ; 95 \%$ CI $-14.0 ;-10.1)$. SIR of robot-assisted hysterectomy increased 30-fold, from 0.4 per 100,000 personyears in 2008 to 11.9 per 100,000 person-years in 2015 (AAPC 44.8; 95\% CI 18.5; 76.8), and SIR of laparoscopic hysterectomy increased 7-fold, from 15.0 per 100,000 person-years in 2000 to 109.7 per 100,000 person-years in 2015 (AAPC 20.4, 95\% CI 15.4; 25.5) (Figure 2).

With respect to hysterectomy due to malignant disease, abdominal hysterectomy declined by $60 \%$, from 66.6 per 100,000 person-years in 2000 to 26.9 per 100,000 personyears in 2015 (AAPC -5.0, 95\% CI -6.9; -3.0). In contrast, SIR of laparoscopic hysterectomy increased 12-fold, from 2.0 per 100,000 person-years to 23.1 per 100,000 person-years (AAPC 23.8, 95\% CI 17.4; 30.6), and robotassisted hysterectomy increased 185 -fold, from 0.2 per 100,000 person-years in 2008 to 37.0 per 100,000 personyears in 2015 (AAPC 42.1, 95\% CI 19.8; 68.5).

\section{Discussion}

In the present study, we found an age-standardized, hysterectomy-corrected incidence rate of hysterectomy of 


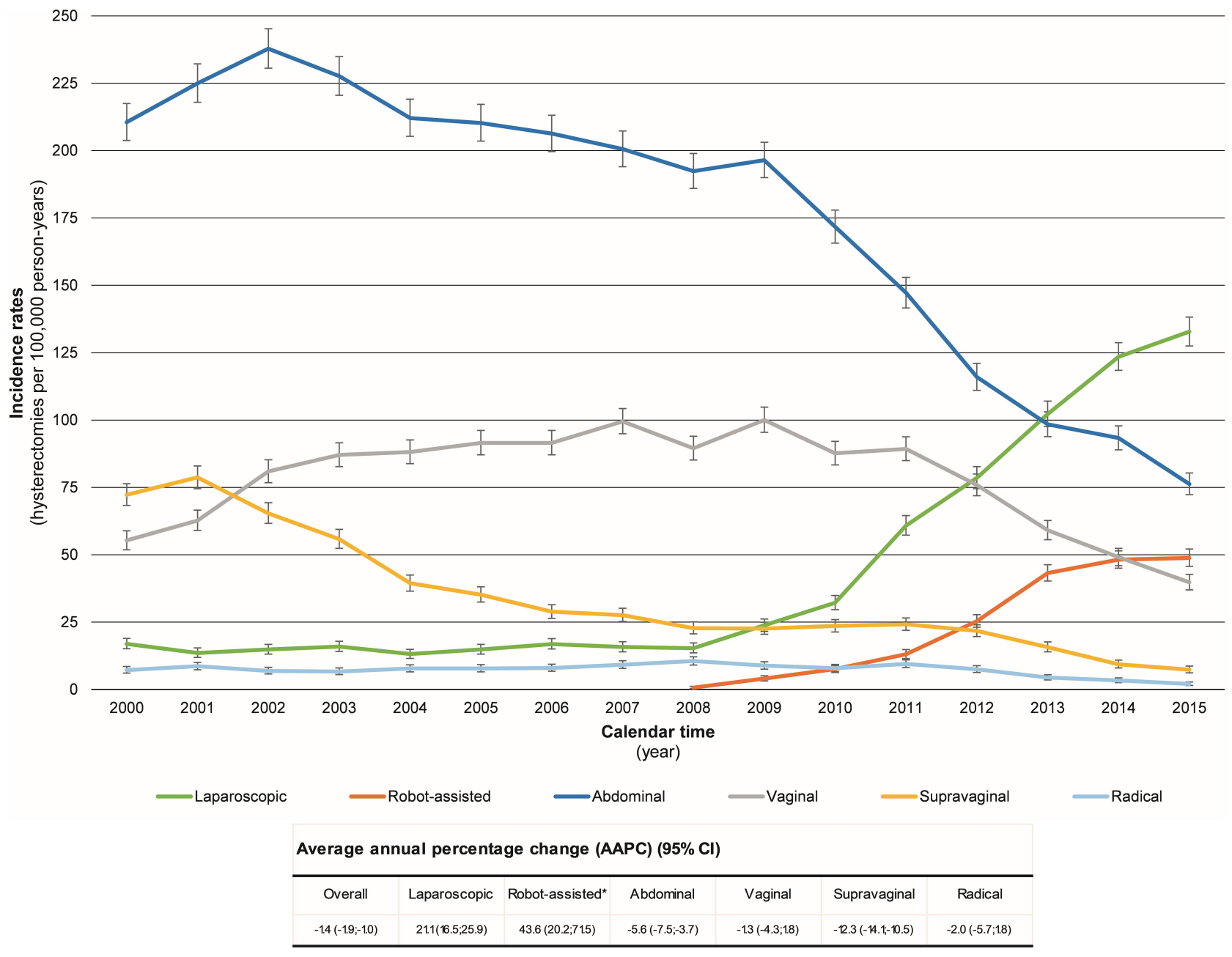

Figure I Age-standardized (US 2010 female population) hysterectomy-corrected incidence rates of hysterectomy over calendar time and corresponding average annual percentage change stratified by surgical procedure.

Note: *Based on SIR from 2008 to 2015 since no robot-assisted hysterectomy was performed prior to 2008.

351.1 per 100,000 person-years. We observed a decline in SIR of hysterectomy during the study period, which was driven by a decline in SIR of benign hysterectomy. Of note, regardless of indication, SIR of abdominal hysterectomy declined substantially over calendar time, while minimally invasive procedures like laparoscopic hysterectomy and robot-assisted hysterectomy increased, surpassing the SIR of abdominal hysterectomy at the end of our study period.

\section{Overall Hysterectomy Incidence Rate}

In general, hysterectomy incidence rates in our study were comparable to results reported in the Nordic countries, but lower than rates in the UK, Germany, US, and Australia. $^{2,12,13,21,25}$ Our findings of a temporal decline in hysterectomy incidence rates are consistent with previous US, Australian, and European studies. ${ }^{5,12,13,25,26}$ However, most of the previous studies reported incidence rates of hysterectomy without correction for previous hysterectomy. Furthermore, the study populations differ by age of women included and by indication for hysterectomy, with most studies reporting incidence rates of benign hysterectomy. Consequently, it is difficult to perform a meaningful comparison of hysterectomy incidence rates across countries. Only a few studies have reported hysterectomy incidence rates after correcting for hysterectomy and reported similar findings as in our study. However, these studies corrected for hysterectomy using data on hysterectomy status derived from surveys and are therefore subject to recall bias. ${ }^{13,21}$

\section{Hysterectomy Due to Benign Disease}

The observed overall decline was a result of declining incidence rates of benign hysterectomy, as reported in previous studies. ${ }^{5,12,26,27}$ This decline may be explained 


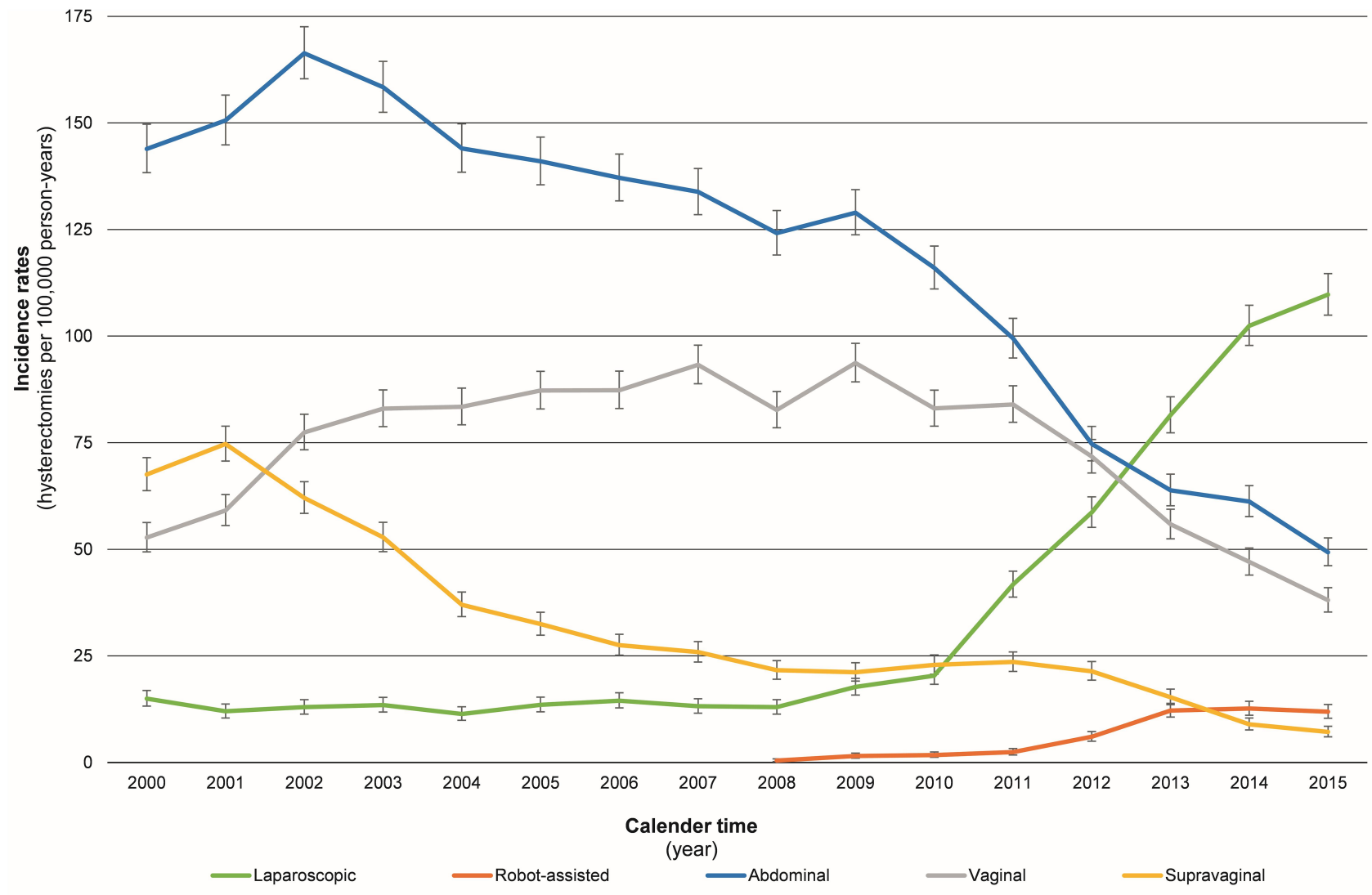

Average annual percentage change (AAPC) $(95 \% \mathrm{CI})$

\begin{tabular}{|c|c|c|c|c|c|}
\hline Benign, overall & Laparoscopic & Robot-assisted & Abdominal & Vaginal & Supravaginal \\
\hline$-2.1(-2.7 ;-16)$ & $20.4(15.4 ; 25.5)$ & $44.8(18.5 ; 76.8)$ & $-5.9(-7.8 ;-4.0)$ & $-14(-4.3 ; 17)$ & $-12.1(-1.0 ;-10.1)$ \\
\hline
\end{tabular}

Figure 2 Age-standardized (US 2010 female population) hysterectomy-corrected incidence rates of hysterectomy over calendar time and corresponding average annual percentage change among women with benign disease, stratified by surgical procedure.

Note: *Based on SIR from 2008 to 2015 since no robot-assisted hysterectomy was performed prior to 2008.

by the introduction of conservative treatment options for fibromas and abnormal uterine bleeding, such as hormonal intrauterine device, ablation, and hysteroscopic resection of the endometrium and/or fibromas. By opting for conservative treatment, women avoid potential surgical complications like bleeding, infection, and damage to the bladder and bowels, ${ }^{6}$ and long-term risks, such as physical and mental impairment, ${ }^{7,10}$ post-surgical pain, ${ }^{8,9}$ and cardiovascular disease. ${ }^{10,11}$ On the other hand, an increasing number of women will have an intact cervix and uterine corpus and will therefore be at risk of developing cervical and endometrial cancer in the future. ${ }^{2,16,28-30}$ Thus, a recent modeling study has projected an increase in cervical cancer incidence rates in the US in the future, particularly at older ages, after considering temporal changes in cervix-removing hysterectomy. ${ }^{31}$

\section{Hysterectomy Due to Malignant Disease}

Incidence rates of hysterectomy due to malignant disease not only reflect incidence rates of gynecological cancers, such as cervical and endometrial cancer, but also the stage of disease. When diagnosed at an early stage, hysterectomy is a crucial part of the treatment. ${ }^{32,33}$ In our sensitivity analysis, where we classified premalignant diagnosis codes as benign, we found that the previously observed temporal increase in the incidence rate of malignant hysterectomy disappeared. This might imply that the previously observed increase in hysterectomy incidence rates when combining premalignant and malignant diagnosis codes was primarily driven by an increase in hysterectomy due to premalignant disease.

\section{Temporal Changes in Type of Procedure}

We found a shift in surgical procedures over time, with abdominal hysterectomy being the most common procedure 
90

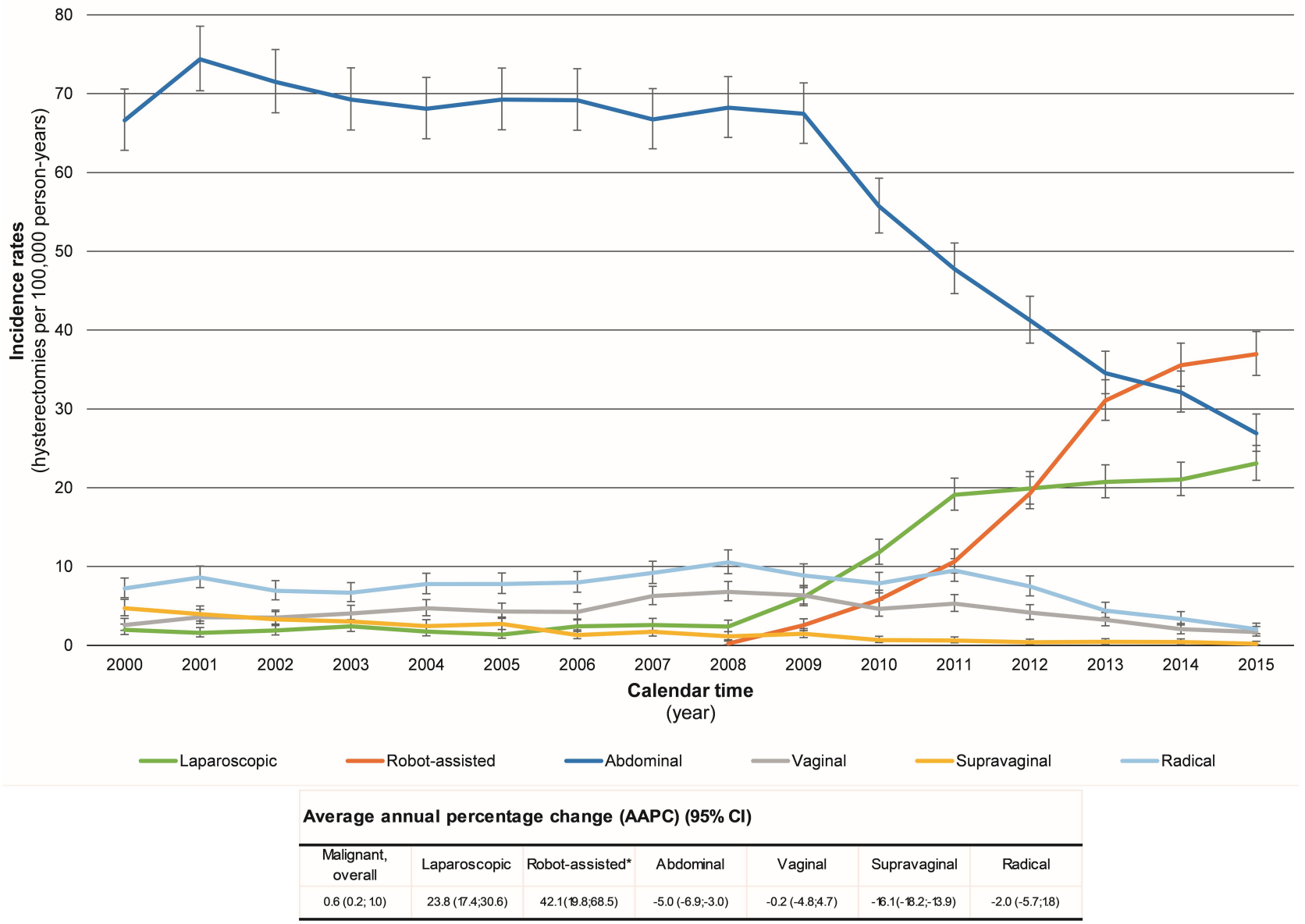

Figure 3 Age-standardized (US 2010 female population) hysterectomy-corrected incidence rates of hysterectomy over calendar time and corresponding average annual percentage change among women with malignant disease, stratified by surgical procedure.

Note: *Based on SIR from 2008 to 2015 since no robot-assisted hysterectomy was performed prior to 2008.

during 2000-2012 and minimally invasive hysterectomy being the leading procedure since 2013 . Our findings, showing a rise in minimally invasive procedures over time, are consistent with previous findings in the same study period. ${ }^{5,13,14,27}$ Studies from Australia, ${ }^{13}$ the US, ${ }^{12}$ and Finland $^{27}$ showed a decline in rates of abdominal hysterectomies and a rise in rates of minimal invasive procedures. However, in the Australian and the US studies, abdominal hysterectomy was still the most common procedure.

When choosing a surgical procedure, it is important to consider the patient's comorbidity (BMI, diabetes, smoking status, medical illnesses, American Society of Anesthesiologists' (ASA) classification of Physical Health, etc.), history of abdominal or vaginal surgery, and the indication. Although complications following benign hysterectomy are rare, several studies show that laparoscopic hysterectomy is superior with respect to risk of bleeding, thromboembolic events, infection, and quality of life. ${ }^{6,26,34-37}$ Minimally invasive procedures may be performed as an outpatient procedure, thereby reducing the length of hospital stay and lowering the costs. $^{26,35,38-40}$ On the other hand, the learning curve for laparoscopic hysterectomy is steep, and studies suggest that the risk of bowel and ureter injuries is higher in laparoscopic hysterectomy compared to abdominal hysterectomy. ${ }^{6,41,42}$ Currently, robot-assisted hysterectomy is comparable to laparoscopic hysterectomy regarding complications and operating time, ${ }^{43}$ albeit the costs associated with robot-assisted hysterectomy are higher. ${ }^{44}$

With regard to hysterectomy performed due to malignant disease, it has recently been questioned whether minimally invasive procedure is the correct choice of procedure for cervical cancer, as studies report that minimally invasive radical hysterectomy is 
associated with shorter disease-free survival and higher mortality compared to laparotomy. ${ }^{32,45}$ However, with respect to surgical treatment for endometrial cancer, no difference in disease-free survival has been observed when comparing robot-assisted and abdominal hysterectomy. ${ }^{33}$

\section{Strengths and Limitations}

When interpreting our results, some limitations should be taken into consideration. Since DNRP was established in 1977, we were unable to retrieve data on hysterectomies performed prior to this date. Instead, we had to rely on a modelling approach to estimate the cumulative lifetime probability of hysterectomy for the hysterectomy correction. As described in previous studies, this method has been found to be very robust. ${ }^{16,18}$ Given that hysterectomy incidence rates and type of procedure vary across countries, our results may not be generalizable to other populations or countries. In our indication and procedure-stratified analyses, we excluded 78 benign radical hysterectomies due to the risk of misclassification. Radical hysterectomy includes the removal of the parametrium on both sides and the upper part of the vagina in addition to the corpus and cervix uteri, and is usually performed in patients with cervical cancer. Unfortunately, we were unable to determine whether they were misclassified by procedure or indication. However, as the number of benign radical hysterectomy accounted for less than $0.1 \%$ of all hysterectomies in the study period, this potential misclassification has minimal impact on our findings.

The strength of this study is the nationwide and population-based design in a tax-funded health care system with virtually complete follow-up. ${ }^{22}$ The use of surgical procedure codes from DNRP to estimate hysterectomy prevalence, instead of relying on survey data as reported in previous studies, ${ }^{13,21}$ reduces the risk of recall bias in our study. Moreover, we were able to collect information on indication and surgical procedure at an individual level, allowing us to perform a sensitivity analysis where we classified premalignant diagnoses as malignant. Our rationale was that these diagnoses are associated with increased risk of cancer and, therefore, are managed differently. Finally, correcting for hysterectomy, so that only women with an intact uterus contributed time at-risk during the study period, resulted in more accurate hysterectomy incidence rates.

\section{Conclusions}

Hysterectomy-corrected SIR of hysterectomy declined from 2000 to 2015, which was driven by declining SIR of benign hysterectomy. Irrespective of indication, the surgical procedure shifted from abdominal hysterectomy to minimally invasive surgical procedures during our study period.

\section{Abbreviations}

AAPC, average annual percentage change; CPR-number, civil registration number; DNRP, Danish National Registry of Patients; IQR, interquartile range; IR, incidence rate; SIR, standardized incidence rate.

\section{Data Sharing Statement}

Restrictions apply for the availability of these data, which were used under the license of this study. Data are available from the authors with the permission of the Danish Data Protection Agency.

\section{Ethical Approval and Informed Consent}

This study was approved by the Danish Data Protection Agency (record number 1-16-02-150-17, approved April 24, 2017). According to the Danish legislation, approval from the Ethics Committee is not required for registry-based research.

\section{Author Contributions}

All authors made substantial contributions to conception and design, acquisition of data, or analysis and interpretation of data; took part in drafting the article or revising it critically for important intellectual content; agreed to submit to the current journal; gave final approval for the version to be published; and agreed to be accountable for all aspects of the work.

\section{Disclosure}

AH has received a speaker's fee from Astra Zeneca, Denmark, outside the submitted work; reports grants from Danish Cancer Society, during the conduct of the study. The remaining authors declare no financial or personal conflict of interest.

\section{References}

1. Evans EC, Matteson KA, Orejuela FJ, et al. Salpingo-oophorectomy at the time of benign hysterectomy: a systematic review. Obstet Gynecol. 2016;128(3):476-485. doi:10.1097/AOG.0000000000001592 
2. Hammer A, Rositch AF, Kahlert J, Gravitt PE, Blaakaer J, Søgaard M. Global epidemiology of hysterectomy: possible impact on gynecological cancer rates. Am J Obstet Gynecol. 2015;213 (1):23-29. doi:10.1016/j.ajog.2015.02.019

3. Mikhail E, Salemi JL, Mogos MF, Hart S, Salihu HM, Imudia AN. National trends of adnexal surgeries at the time of hysterectomy for benign indication, United States, 1998-2011. Am J Obstet Gynecol. 2015;213(5):713.e1-713.e13. doi:10.1016/j.ajog.2015.04.031

4. Moss EL, Morgan G, Martin AP, Sarhanis P, Ind T. Surgical trends, outcomes and disparities in minimal invasive surgery for patients with endometrial cancer in England: a retrospective cohort study. BMJ Open. 2020;10(9):e036222. doi:10.1136/bmjopen-2019-036222

5. Lykke R, Blaakær J, Ottesen B, Gimbel H. Hysterectomy in Denmark 1977-2011: changes in rate, indications, and hospitalization. Eur J Obstet Gynecol Reprod Biol. 2013;171(2):333-338. doi:10.1016/j. ejogrb.2013.09.011

6. Brummer THI, Jalkanen J, Fraser J, et al. FINHYST, a prospective study of 5279 hysterectomies: complications and their risk factors. Hum Reprod. 2011;26(7):1741-1751. doi:10. 1093/humrep/der116

7. Wilson LF, Pandeya N, Byles J, Mishra GD. Hysterectomy and perceived physical function in middle-aged Australian women: a 20-year population-based prospective cohort study. Qual Life Res. 2018;27(6):1501-1511. doi:10.1007/s11136-018-1812-9

8. Brandsborg B, Dueholm M, Nikolajsen L, Kehlet H, Jensen TS. Prospective study of risk factors for pain persisting. Clin J Pain. 2009;25(4):263-268. doi:10.1097/AJP.0b013e31819655ca

9. Berner E, Qvigstad E, Myrvold AK, Lieng M. Pelvic pain and patient satisfaction after laparoscopic supracervical hysterectomy: prospective trial. J Minim Invasive Gynecol. 2014;21(3):406-411. doi:10.1016/j.jmig.2013.10.011

10. Wilson L, Pandeya N, Byles J, Mishra G. Hysterectomy and incidence of depressive symptoms in midlife women: the Australian longitudinal study on women's health. Epidemiol Psychiatr Sci. 2018;27(4):381-392. doi:10.1017/S2045796016001220

11. Ingelsson E, Lundholm C, Johansson ALV, Altman D. Hysterectomy and risk of cardiovascular disease: a population-based cohort study. Eur Heart J. 2011;32(6):745-750. doi:10.1093/eurheartj/ehq477

12. Wright JD, Herzog TJ, Tsui J, et al. Nationwide trends in the performance of inpatient hysterectomy in the United States. Obstet Gynecol. 2013;122(2 Pt 1):233-241. doi:10.1097/AOG.0b013e31 $8299 \mathrm{a} 6 \mathrm{cf}$

13. Wilson LF, Pandeya N, Mishra GD. Hysterectomy trends in Australia, 2000-2001 to 2013-2014: joinpoint regression analysis. Acta Obstet Gynecol Scand. 2017;96(10):1170-1179. doi:10.1111/ aogs. 13182

14. Lundholm C, Forsgren C, Johansson ALV, Cnattingius S, Altman D. Hysterectomy on benign indications in Sweden 1987-2003: a nationwide trend analysis. Acta Obstet Gynecol Scand. 2009;88 (1):52-58. doi:10.1080/00016340802596017

15. Rositch AF, Nowak RG, Gravitt PE. Increased age and race-specific incidence of cervical cancer after correction for hysterectomy prevalence in the United States from 2000 to 2009. Cancer. 2014;120 (13):2032-2038. doi:10.1002/cncr.28548

16. Hammer A, Kahlert J, Rositch A, et al. The temporal and age-dependent patterns of hysterectomy-corrected cervical cancer incidence rates in Denmark: a population-based cohort study. Acta Obstet Gynecol Scand. 2017;96(2):150-157. doi:10.1111/aogs.13057

17. Gustafson LW, Booth BB, Kahlert J, et al. Trends in hysterectomy-corrected uterine cancer mortality rates during 2002 to 2015: mortality of nonendometrioid cancer on the rise? Int J Cancer. 2021;148:584-592.

18. Hammer A, Kahlert J, Gravitt PE, Rositch AF. Hysterectomycorrected cervical cancer mortality rates in Denmark during 2002-2015: a registry-based cohort study. Acta Obstet Gynecol Scand. 2019;98(8):1063-1069. doi:10.1111/aogs.13608
19. Beavis AL, Gravitt PE, Rositch AF. Hysterectomy-corrected cervical cancer mortality rates reveal a larger racial disparity in the United States. Cancer. 2017;123(6):1044-1050. doi:10.1002/cncr.30507

20. Stang A. Impact of hysterectomy on the age-specific incidence of cervical and uterine cancer in Germany and other countries. Eur J Public Health. 2013;23(5):879-883. doi:10.1093/eurpub/cks080

21. Stang A, Merrill RM, Kuss O. Prevalence-corrected hysterectomy rates by age and indication in Germany 2005-2006. Arch Gynecol Obstet. 2012;286(5):1193-1200. doi:10.1007/s00404-012-2415-2

22. Schmidt M, Pedersen L, Sørensen HT. The Danish civil registration system as a tool in epidemiology. Eur J Epidemiol. 2014;29:541-549. doi:10.1007/s10654-014-9930-3

23. Schmidt M, Schmidt SAJ, Sandegaard JL, Ehrenstein V, Pedersen L, Sørensen HT. The Danish national patient registry: a review of content, data quality, and research potential. Clin Epidemiol. 2015;7:449-490. doi:10.2147/CLEP.S91125

24. Kim HJ, Fay MP, Feuer EJ, Midthune DN. Permutation tests for joinpoint regression with applications to cancer rates. Stat Med. 2000;19(3):335-351. doi:10.1002/(SICI)1097-0258(20000215) 19:3<335::AID-SIM336>3.0.CO;2-Z

25. Doll KM, Dusetzina SB, Robinson W. Trends in inpatient and outpatient hysterectomy and oophorectomy rates among commercially insured women in the United States, 2000-2014. JAMA Surg. 2016;151(9):876-877. doi:10.1001/jamasurg.2016.0804

26. Settnes A, Topsoee MF, Moeller C, et al. Reduced complications following implementation of laparoscopic hysterectomy: a Danish population-based cohort study of minimally invasive benign gynecologic surgery between 2004 and 2018. J Minim Invasive Gynecol. 2020;27(6):1344-1353.e3. doi:10.1016/j.jmig.2019.11.005

27. Mäkinen J, Brummer T, Jalkanen J, et al. Ten years of progress Improved hysterectomy outcomes in Finland 1996-2006: a longitudinal observation study. BMJ Open. 2013;3(10):1-8. doi:10.1136/bmjopen-2013-003169

28. Ruiz G, Unzurrunzaga DA, Brewster DH, Wild SH. Declining hysterectomy prevalence and the estimated impact on uterine cancer incidence in Scotland. Cancer Epidemiol. 2019;59:227-231. doi:10.1016/j.canep.2019.02.008

29. Rahib L, Smith BD, Aizenberg R, Rosenzweig AB, Fleshman JM, Matrisian LM. Projecting cancer incidence and deaths to 2030: the unexpected burden of thyroid, liver, and pancreas cancers in the United States. Cancer Res. 2014;74(11):2913-2921. doi:10.1158/ 0008-5472.CAN-14-0155

30. Temkin SM, Minasian L, Noone AM. The end of the hysterectomy epidemic and endometrial cancer incidence: what are the unintended consequences of declining hysterectomy rates? Front Oncol. 2016;6:1-6. doi:10.3389/fonc.2016.00089

31. Simms KT, Yuill S, Killen J, et al. Historical and projected hysterectomy rates in the USA: implications for future observed cervical cancer rates and evaluating prevention interventions. Gynecol Oncol. 2020;158(3):710-718. doi:10.1016/j.ygyno.2020.05.030

32. Ramirez PT, Frumovitz M, Pareja R, et al. Minimally invasive versus abdominal radical hysterectomy for cervical cancer. $N$ Engl J Med. 2018;379(20):1895-1904. doi:10.1056/NEJMoa1806395

33. Janda M, Gebski V, Davies LC, et al. Effect of total laparoscopic hysterectomy vs total abdominal hysterectomy on disease-free survival among women with stage $\mathrm{i}$ endometrial cancer: a randomized clinical trial. JAMA - J Am Med Assoc. 2017;317(12):1224-1233. doi:10.1001/jama.2017.2068

34. Wiser A, Holcroft CA, Tulandi T, Abenhaim HA. Abdominal versus laparoscopic hysterectomies for benign diseases: evaluation of morbidity and mortality among 465,798 cases. Gynecol Surg. 2013;10 (2):117-122. doi:10.1007/s10397-013-0781-9

35. Mallick R, English J, Waters N. Total laparoscopic hysterectomy versus total abdominal hysterectomy in the treatment of benign gynaecological disease: a retrospective review over 5 years. Obstet Gynecol Int J. 2016;5(7). doi:10.15406/ogij.2016.05.00186 
36. Nieboer TE, Hendriks JCM, Bongers MY, Vierhout ME, Kluivers KB. Quality of life after laparoscopic and abdominal hysterectomy: a randomized controlled trial. Obstet Gynecol. 2012;119 (1):85-91. doi:10.1097/AOG.0b013e31823d3b00

37. Jørgensen SL, Mogensen O, Wu C, et al. Nationwide introduction of minimally invasive robotic surgery for early-stage endometrial cancer and its association with severe complications. JAMA Surg. 2019;154 (6):530-538. doi:10.1001/jamasurg.2018.5840

38. Warren L, Ladapo JA, Borah BJ, Gunnarsson CL. Open abdominal versus laparoscopic and vaginal hysterectomy: analysis of a large United States payer measuring quality and cost of care. J Minim Invasive Gynecol. 2009;16(5):581-588. doi:10.1016/j.jmig.2009. 06.018

39. Christiansen UJ, Kruse AR, Olesen PG, Lauszus FF, Kesmodel US, Forman A. Outpatient vs inpatient total laparoscopic hysterectomy: a randomized controlled trial. Acta Obstet Gynecol Scand. 2019;98 (11):1420-1428. doi:10.1111/aogs.13670

40. Brummer THI, Jalkanen J, Fraser J, et al. FINHYST 2006 - National prospective 1-year survey of 5279 hysterectomies. Hum Reprod. 2009;24(10):2515-2522. doi:10.1093/humrep/dep229
41. Brummer THI, Seppälä TT, Härkki PSM. National learning curve for laparoscopic hysterectomy and trends in hysterectomy in Finland 2000-2005. Hum Reprod. 2008;23(4):840-845. doi:10.1093/hum$\mathrm{rep} / \mathrm{den} 006$

42. Johnson N, Barlow D, Lethaby A, Tavender E, Curr L, Garry R. Methods of hysterectomy: systematic review and meta-analysis of randomised controlled trials. BMJ. 2005;25:330.

43. Albright BB, Witte $\mathrm{T}$, Tofte $\mathrm{AN}$, et al. Robotic versus laparoscopic hysterectomy for benign disease: a systematic review and meta-analysis of randomized trials. J Minim Invasive Gynecol. 2016;23(1):18-27. doi:10.1016/j.jmig.2015.08.003

44. Lönnerfors C, Reynisson P, Persson J. A randomized trial comparing vaginal and laparoscopic hysterectomy vs robot-assisted hysterectomy. J Minim Invasive Gynecol. 2015;22(1):78-86. doi:10.1016/j.jmig.2014.07.010

45. Melamed A, Margul DJ, Chen L, et al. Survival after minimally invasive radical hysterectomy for early-stage cervical cancer. $N$ Engl J Med. 2018;379(20):1905-1914. doi:10.1056/NEJMoa 1804923
Clinical Epidemiology

\section{Publish your work in this journal}

Clinical Epidemiology is an international, peer-reviewed, open access, online journal focusing on disease and drug epidemiology, identification of risk factors and screening procedures to develop optimal preventative initiatives and programs. Specific topics include: diagnosis, prognosis, treatment, screening, prevention, risk factor modification, systematic reviews, risk \& safety of medical interventions, epidemiology \& biostatistical methods, and evaluation of guidelines, translational medicine, health policies \& economic evaluations. The manuscript management system is completely online and includes a very quick and fair peer-review system, which is all easy to use. 